Article

\title{
Surface Modification of Bombyx mori Silk Fibroin Film via Thiol-Ene Click Chemistry
}

\author{
Xiaoning Zhang ${ }^{1, * \mathbb{C}}$, Jianwei Liang ${ }^{1}$, Zhenyu Chen ${ }^{1}$, Carrie Donley ${ }^{2}$, Xiaolin Zhang ${ }^{1}$ and \\ Guotao Cheng 1 \\ 1 State Key Laboratory of Silkworm Genome Biology, College of Biotechnology, Southwest University, \\ Chongqing 400715, China; kubf@email.swu.edu.cn (J.L.); c618@email.swu.edu.cn (Z.C.); \\ zx11362969@163.com (X.Z.); cheng_2001@swu.edu.cn (G.C.) \\ 2 Chapel Hill Analytical and Nanofabrication Laboratory, Department of Applied Physical Sciences, \\ University of North Carolina at Chapel Hill, Chapel Hill, NC 27599-3216, USA; cdonley@email.unc.edu \\ * Correspondence: xzhang@swu.edu.cn; Tel.: +86-159-2281-5612
}

Received: 26 March 2020; Accepted: 21 April 2020; Published: 25 April 2020

check for updates

\begin{abstract}
The purpose of this work is to develop a strategy for the surface modification of Bombyx mori silk fibroin (SF) film, via a thiol-ene click reaction, in order to expand its potential applicability. To achieve this goal, terminal thiol groups, from reduced glutathione, were introduced onto the surface of the SF film via a carbodiimide coupling reaction. These immobilized thiol groups act as robust crosslinkers and allow us to rapidly functionalize the surface with alkene group-containing molecules via ultraviolet (UV) light-initiated thiol-ene click chemistry. The X-ray photoelectron spectroscopy results specifically determined that alkene group-containing molecules and UV illumination are required for the thiol-ene click modification to take place on thiolated SF films. Fourier-transform infrared spectroscopy characterization of SF films indicated that the $\beta$-sheet conformation of SF was hampered throughout the modification process. Furthermore, it was found that the processes for thiol-ene click chemistry that took place on the SF film surface did not affect the thermal stability of SF films significantly, indicating the developed strategy preserved the intrinsic properties of the SF film. Taking into account that many alkene-containing molecules are suitable for thiol-ene click chemistry, we see immense potential for the incorporation of SF films into various applications, using this methodology.
\end{abstract}

Keywords: Bombyx mori; silk fibroin film; thiol-ene click chemistry; surface modification

\section{Introduction}

As an ancient material, the degummed silk fibroin (SF) fibers can be dissolved in aqueous $\mathrm{CaCl}_{2}$-ethanol- $\mathrm{H}_{2} \mathrm{O}$ solution (1:2:8 in molar ratio). After desalination, the regenerated silk fibroin solutions can be processed into different forms such as gels, sponges and films [1,2]. Among those, regenerated silk fibroin film shows particular promising features due to its optical transparency, biocompatibility, mechanical robustness and flexible nature [3]. Therefore, SF film is considered well suited to serve as a platform for conformal devices, and can be easily applied to curved objects and adhere conformally [4]. In addition, it is known that the application of silk fibroin films can be expanded with the proper surface modification $[5,6]$.

As one of the methods for material modification, click chemistry is appealing because it is efficient yet stereospecific, high-yielding with minimal byproducts, and environmentally-friendly. Besides, click chemistry can take place under aqueous conditions and requires simple reaction conditions-it does not need extremely high temperature or pressure, and is $\mathrm{pH}$ insensitive. Thus, click chemistry has become a powerful tool in the field of biomaterial labeling and modification [7]. 
Using click chemistry, surface modification of silk fibroin film can be achieved, and the majority of studies focus on Cu-catalyzed azide-alkyne cycloaddition reactions [8,9]. However, azides should be handled with extra care, as both organic and inorganic azides are potentially explosive substances [10].

As one type of click chemistry, ultraviolet (UV) light-initiated thiol-ene click chemistry involves the addition reaction between the thiol radicals and the carbon-carbon double bonds in a regio- and enantioselective way [11]. Although the report is limited, recent exploration has demonstrated that thiol-ene click reactions can be used for the formation of an SF-based hydrogel network [12]. In their work, alkene groups (from carbic anhydride) were first incorporated onto the SF molecules, followed by thiol-ene photocrosslinking with thiol groups containing molecules. The procedure of their work required a certain chemical synthetic process for the reactant preparation, and carbic anhydride exhibits unfavorable biocompatibility.

In this work, by adopting a different strategy, we installed the thiol groups (from reduced glutathione (GSH)) onto the silk fibroin film, so that they could act as specific sites for the integration of functional groups from other molecules via a thiol-ene click reaction. We characterized silk fibroin films throughout the reaction processes and demonstrated the successful attachment of alkene-containing molecules onto the thiol-terminated silk fibroin films. Through the developed strategy, molecules with specific functionalities can be introduced onto the SF film. In addition, as thiol-ene click chemistry is photoinitiated, the developed strategy also demonstrates great potential in constructing photolithographic patterning on silk fibroin film, which therefore expands the potential applicability of the SF film in its device-based construction.

\section{Materials and Methods}

\subsection{Materials}

Cocoons of silkworm Bombyx mori (a Chinese strain demoted as 872) were obtained from the College of Biotechnology, Southwest University, China. $\mathrm{Na}_{2} \mathrm{CO}_{3}$ and $\mathrm{Na}_{2} \mathrm{HPO}_{4}$ were bought from KeLong Chemical Reagent Co., Ltd. (Chengdu, Sichuan, China). $\mathrm{NaH}_{2} \mathrm{PO}_{4} \bullet 2 \mathrm{H}_{2} \mathrm{O}$ and $\mathrm{CaCl}_{2}$ were bought from Yuanye Bio-Technology Co., Ltd. (Tianjin, China). $\mathrm{C}_{2} \mathrm{H}_{5} \mathrm{OH}$ was bought from Chuandong Chemical Co., Ltd. (Chongqing, China). Reduced glutathione (GSH) and N-hydroxysuccinimide (NHS) were bought from Solarbio Co., Ltd. (Beijing, China). 1-(3-Dimethylaminopropyl)-3-ethylcarbodiimide hydrochloride $\left(\mathrm{EDC} \bullet \mathrm{HCl}\right.$ ) was bought from Adamas-beta (Shanghai, China). $\mathrm{H}_{2} \mathrm{C}=\mathrm{CH}\left(\mathrm{CH}_{2}\right)_{8} \mathrm{CH}_{2} \mathrm{Br}$ (BUD) was bought from Sigma Aldrich (St. Louis, MO, USA). $\mathrm{CH}_{3}\left(\mathrm{CH}_{2}\right)_{9} \mathrm{CH}_{2} \mathrm{Br}$ was bought from HEOWNS (Tianjin, China). All chemicals used were of analytical grade and were used as received without any further purification. The water, with a resistivity greater than $18 \mathrm{M} \Omega \cdot \mathrm{cm}$, used in this work was obtained from an onsite Milli-Q Direct-8 purification system (Millipore, Molsheim, France).

\subsection{Material Characterization}

A Kratos Axis Ultra DLD X-ray Photoelectron Spectrometer (XPS, Kratos Analytical Co., Ltd., Manchester, UK) was used to analyze the surface elemental composition of the samples. Step sizes 1.0 and 0.1 electron volt $(\mathrm{eV})$ were used for survey and high-resolution scans, respectively. The takeoff angle was $0^{\circ}$ from the surface normal. The XPS data were analyzed using Kratos Vision 2.0 software (Kratos Analytical Co., Ltd., Manchester, UK).

Differential scanning calorimetry (DSC) was performed to assess the thermal properties of the SF films (DSC, HSC-3, Beijing HengJiu Scientific Instrument Factory, Beijing, China). The samples were heated at $2{ }^{\circ} \mathrm{C} / \mathrm{min}$ from $30^{\circ} \mathrm{C}$ to $450{ }^{\circ} \mathrm{C}$ with a nitrogen gas flow rate of $50 \mathrm{~mL} \cdot \mathrm{min}^{-1}$. Samples weighing approximately $7 \mathrm{mg}$ were placed in $\mathrm{Al}_{2} \mathrm{O}_{3}$ DSC pans and mounted in the DSC cell. An empty $\mathrm{Al}_{2} \mathrm{O}_{3}$ pan was used as a reference for each measurement.

Static water contact angles of the samples were measured using a home-made goniometer and analyzed by Image J (Java 1.6.0_24) software (National Institute of Health, Bethesda, MD, USA). 
To calculate the contact angle, measurements at three areas on each film, and three films of each sample, were analyzed.

The secondary structure of the silk fibroin was studied by the attenuated total reflection Fourier Transform Infrared Spectroscopy (ATR-FTIR, Nicolet iN10, Thermo Scientific, Waltham, MA, USA), and the spectra were recorded at a resolution of $4 \mathrm{~cm}^{-1}$, from $400 \mathrm{~cm}^{-1}$ to $4000 \mathrm{~cm}^{-1}$, for 32 scans.

\subsection{Covalent Coupling of BUD to SF Films}

The water-insoluble SF films (called WA-SF films) were fabricated from $6.0 \% \mathrm{SF}(w / v)$ solution as previously reported $[13,14]$. The process to graft glutathione (GSH) onto SF film covalently is illustrated in Figure 1. Specifically, the prepared WA-SF films were first soaked in a phosphate-buffered saline (PBS) buffer of $\mathrm{pH} 6.5$ for $30 \mathrm{~min}$, in order to bring hydrophilic functional groups to the film's surface. Then, the films were immersed in an EDC/NHS solution, prepared by dissolving EDC and NHS in a PBS buffer $(\mathrm{pH}=6.5)$ with the final concentration of $0.5 \mathrm{mg} / \mathrm{mL}$ and $0.7 \mathrm{mg} / \mathrm{mL}$ respectively, for $15 \mathrm{~min}$ at room temperature, in order to activate carboxyl groups $(-\mathrm{COOH})$ in the SF. Next, the activated SF films were rinsed with the PBS buffer, and then contacted with $0.1 \mathrm{mg} / \mathrm{mL}$ GSH in the PBS buffer for $2 \mathrm{~h}$, at room temperature. The resulting films (called GSH-SF films) were rinsed with water thoroughly to remove unbound GSH and chemical remains [15].

To functionalize the alkene-containing molecules onto the GSH-SF films through thiol-ene click chemistry, $0.2 \mathrm{~mL}$ of a neat BUD liquid was placed on the surface of each sample (here, the BUD molecule was selected as a model molecule because the terminal bromine atom can act as an elemental tag for XPS detection, which enables us to track the reaction process). The sample was covered by a quartz coverslip ( $0.5 \mathrm{~mm}$ thickness) to achieve an even distribution of the BUD liquid across the sample surface, and was illuminated with a $40 \mathrm{~W}, 365 \mathrm{~nm}$ ultraviolet light-emitting diode (UV-LED) for a certain time. Each sample was placed $20 \mathrm{~mm}$ beneath the UV-LED light source. Samples were then sonicated in ethanol to remove nonspecifically adsorbed BUD molecules, followed by thorough rinsing in deionized water. Prior to characterization, the prepared samples were equilibrated in an environmental chamber at $25^{\circ} \mathrm{C}$ and $60 \%$ relative humidity for $12 \mathrm{~h}$. The prepared BUD-modified SF films (called BUD-SF films) demonstrated excellent transparency.
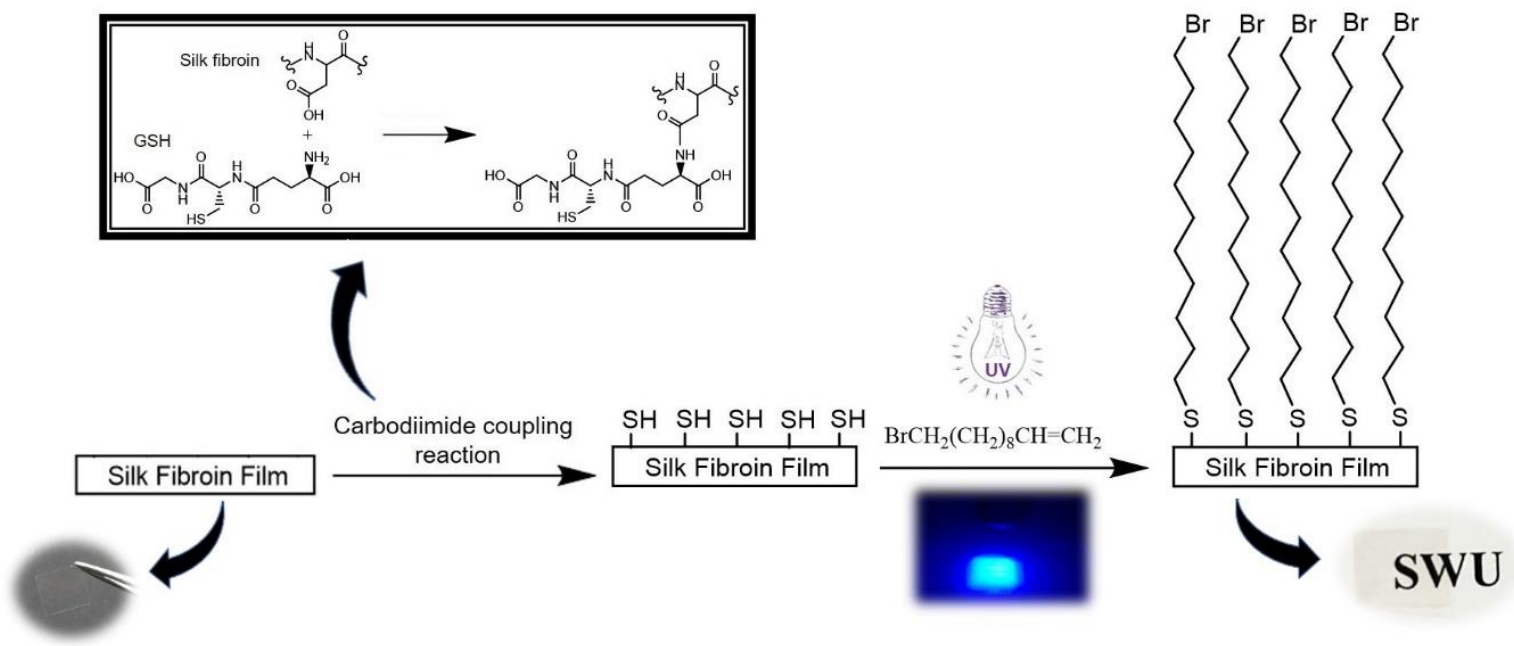

Figure 1. Schematic diagram for the covalent coupling of $\mathrm{H}_{2} \mathrm{C}=\mathrm{CH}\left(\mathrm{CH}_{2}\right)_{8} \mathrm{CH}_{2} \mathrm{Br}$ (BUD) to silk fibroin (SF) films. 


\section{Results}

\subsection{X-ray Photoelectron Spectroscopy Results}

X-ray photoelectron spectroscopy (XPS) measurements were used to investigate the chemistry at the surface of the samples throughout the modification processes. Once GSH-SF films were exposed to neat BUD and illuminated under $365 \mathrm{~nm}$ UV-LED for $10 \mathrm{~min}$, a bromine peak could be identified by XPS (Figure 2a) as a consequence of the thiol-ene click reaction between the GSH-SF films and BUD molecules, with a bromine-to-carbon $(\mathrm{Br} / \mathrm{C})$ ratio of $0.02017 \pm 0.00060$. This indicates that the BUD molecules were grafted onto the GSH-SF films through thiol-ene click chemistry successfully. In addition, when the illumination time was shortened to $5 \mathrm{~min}$, the number of BUD molecules attached to the GSH-SF film decreased dramatically, which is consistent with the previous study, indicating that an introduction period is required for the photoinitiated thiol-ene coupling reaction [16]. When the illumination time was elongated to $60 \mathrm{~min}$, however, the number of BUD molecules attached to the GSH-SF film did not increase significantly. This might be because the yield was limited by the number of thiol groups immobilized on the SF film (Supplementary Material).

We also illuminated GSH-SF films contacted with neat 1-bromoundecane, the analogue of BUD, to compare the differences between it and BUD molecules with regard to surface attachment, and we were unable to detect the element bromine by XPS (Figure $2 b, \mathrm{Br} / \mathrm{C}$ ratio $=0$ ). Therefore, alkene groups are necessary for the attachment of molecules onto a GSH-SF film surface.
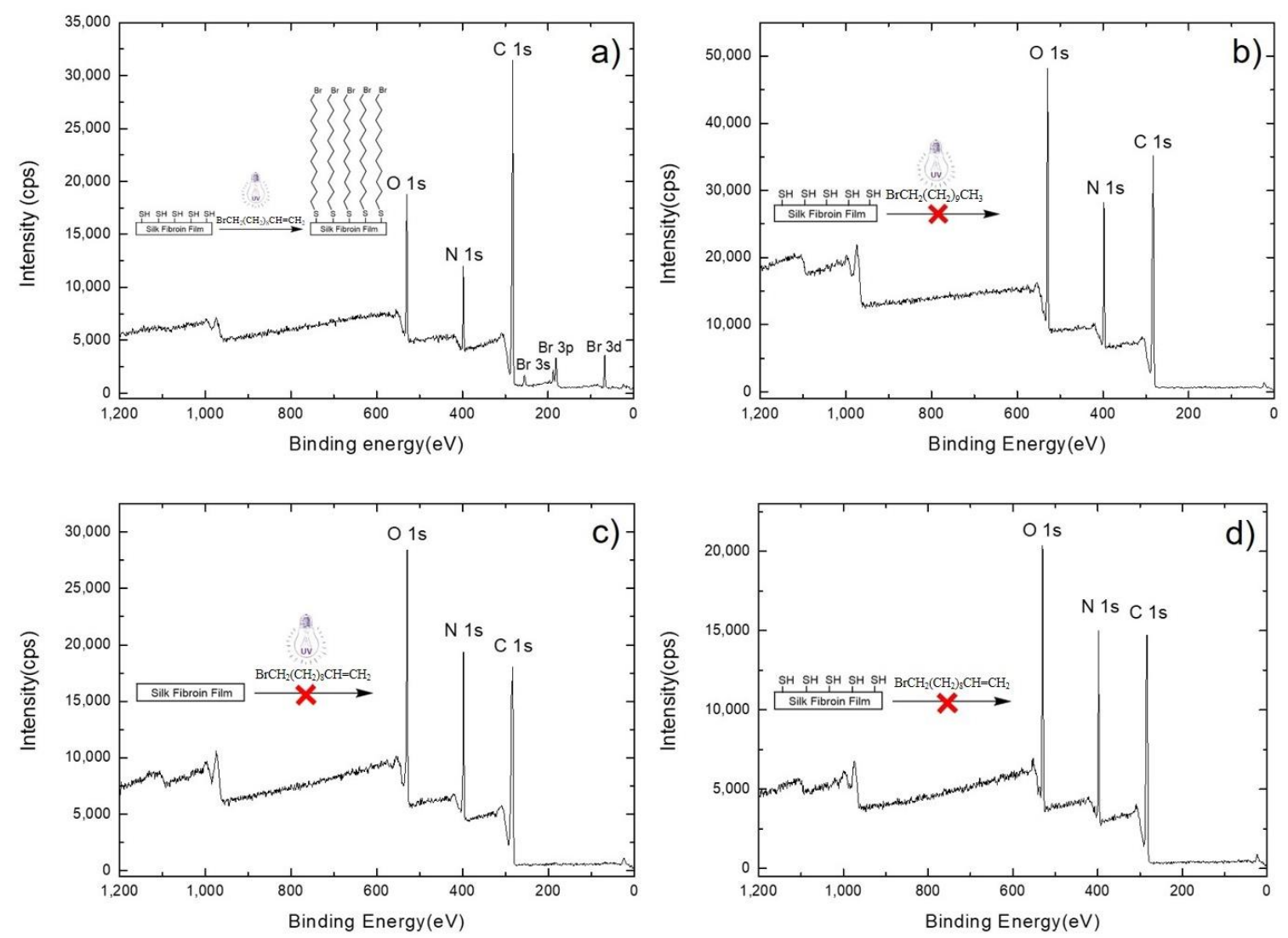

Figure 2. Representative X-ray photoelectron (XP) survey spectra of: (a) Reduced glutathione grafted SF (GSH-SF) film with neat BUD illuminated by $365 \mathrm{~nm}$ ultraviolet light-emitting diode (UV-LED) for $10 \mathrm{~min}$; (b) GSH-SF film with neat 1-bromoundecane illuminated by $365 \mathrm{~nm}$ UV-LED for $10 \mathrm{~min}$; (c) The water-insoluble SF (WA-SF) film with neat BUD illuminated by $365 \mathrm{~nm}$ UV-LED for $10 \mathrm{~min}$; and (d) GSH-SF film with neat BUD in the absence of any UV exposure. The binding energies are assigned as follows: O1s: $532 \mathrm{eV}$; N1s: $400 \mathrm{eV}$; C1s: $285 \mathrm{eV}$; Br3d: $70 \mathrm{eV}$. Each reaction is illustrated schematically in the inset of Figure 2. 
Then, we illuminated WA-SF films with UV in the presence of BUD molecules to determine if BUD attachment was specific to the thiolated SF films. The XPS spectra showed that 10 min of UV illumination did not result in any observable bromine electrons (Figure 2c). The above finding supports the view that the existence of terminal thiol groups on the SF film is critical for the surface attachment of functional molecules through this developed strategy. Furthermore, we placed GSH-modified SF films in contact with BUD molecules, but without UV illumination. After we thoroughly sonicated and rinsed the films, the signal from bromine electrons could not be detected by XPS (Figure 2d), indicating that UV illumination is required for the surface modification of SF films using this developed approach.

\subsection{Differential Scanning Calorimetry Analysis}

Figure 3 illustrates the DSC curves of WA-SF, GSH-SF and BUD-SF films. All curves demonstrated degradation peaks in the region around $270{ }^{\circ} \mathrm{C}$, indicating the surface modification of silk fibroin film through this developed strategy does not significantly affect the thermal stability of the silk fibroin film. The degradation temperatures of samples from each group are listed in Table 1.

Table 1. The degradation temperatures of the silk fibroin (SF) films from each group.

\begin{tabular}{cc}
\hline Group & Degradation Temperatures \\
\hline WA-SF & $269.1 \pm 1.7^{\circ} \mathrm{C}$ \\
GSH-SF & $270.5 \pm 3.5^{\circ} \mathrm{C}$ \\
BUD-SF & $269.7 \pm 4.1^{\circ} \mathrm{C}$ \\
\hline
\end{tabular}

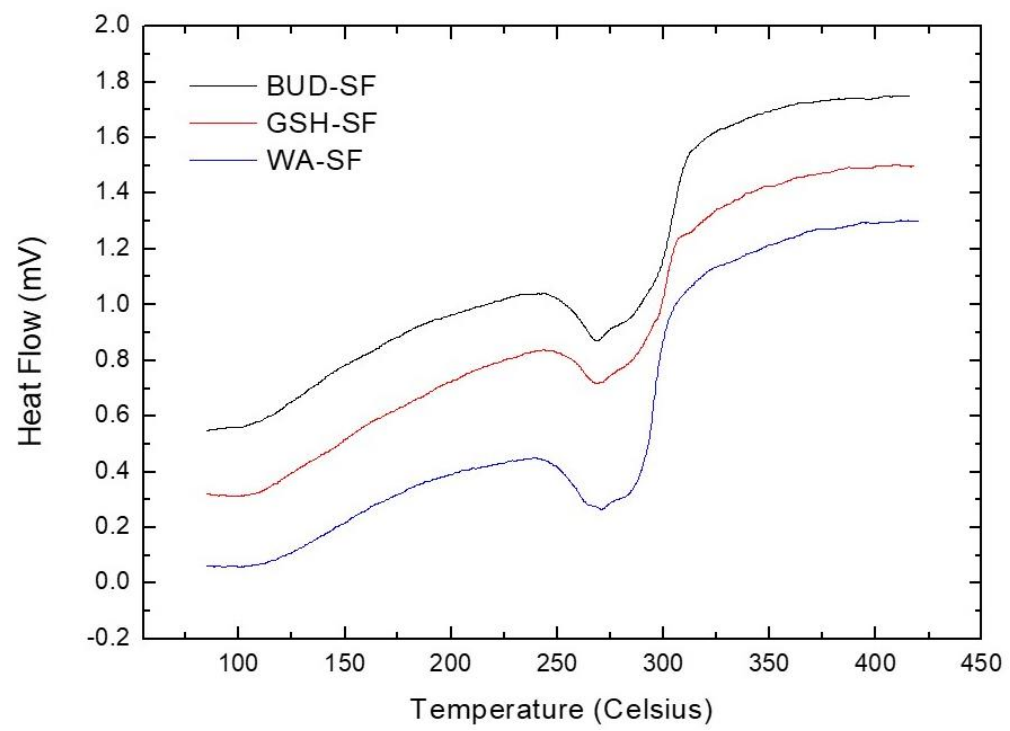

Figure 3. Differential scanning calorimetry (DSC) curves of WA-SF, GSH-SF and BUD-SF films.

\subsection{Static Contact Angle Measurement}

The surface properties of samples have been studied via static contact angle measurements. As GSH acts as a hydrophilic molecule [17], it follows that the GSH-SF films are more hydrophilic compared to WA-SF films. The hydrophobicity of the BUD-SF films increased, and we attribute the increased contact angle to the overall changes in the chemical composition of the surface after the thiol-ene click reaction (the introduction of methylene groups (- $\left.\mathrm{CH}_{2}-\right)$ from BUD molecules). The contact angle values of the films from different groups are listed in Table 2. 
Table 2. Static contact angle values for water droplets on the SF films from each group.

\begin{tabular}{|c|c|}
\hline Group & Contact Angle \\
\hline WA-SF & $55.0 \pm 3.1^{\circ}$ \\
\hline GSH-SF & $49.8 \pm 1.9^{\circ}=$ \\
\hline BUD-SF & $65.5 \pm 3.1^{\circ}$ \\
\hline
\end{tabular}

\subsection{Infrared Spectroscopy Analysis}

FTIR spectroscopy was used to assess changes in the secondary structures of silk fibroin with respect to the post-cast processing steps, including water-annealing, GSH-grafting and BUD-modification via thiol-ene click reaction. Initial examination of the spectral curves (Figure $4 \mathrm{a}$ ) indicates that the FTIR bands for amide I (1600-1700 $\left.\mathrm{cm}^{-1}\right)$ and amide II $\left(1500-1600 \mathrm{~cm}^{-1}\right)$ [18] of all samples are found within the same regions.
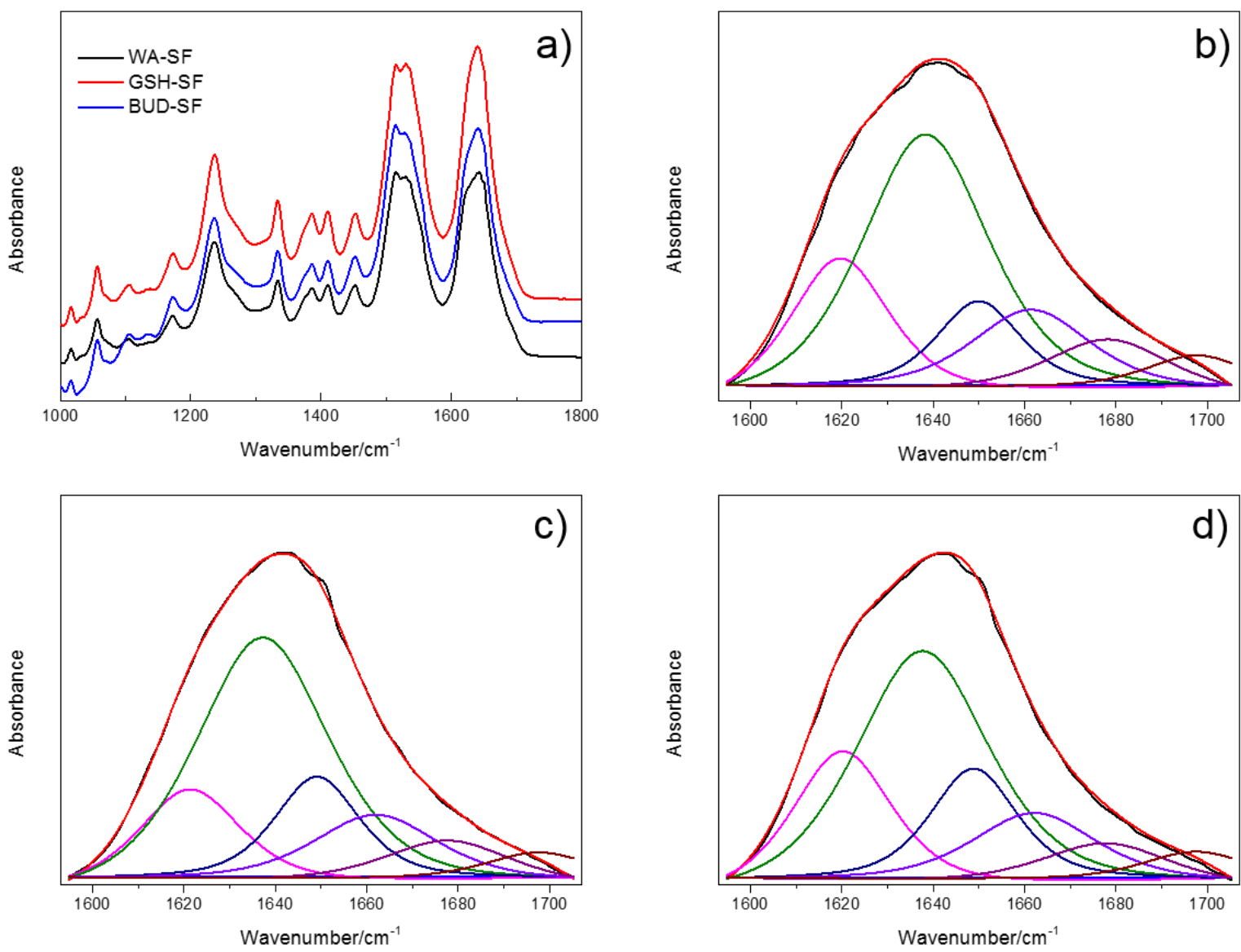

Figure 4. (a) Fourier Transform Infrared Spectroscopy (FTIR) spectra of WA-SF, GSH-SF, and BUD-SF films. Deconvoluted FTIR curves for: (b) WA-SF; (c) GSH-SF; and (d) BUD-SF films. The black curve represents the original FTIR spectrum, the red curve represents the spectral fit. The six deconvoluted curves are shown below the original FTIR spectrum and the spectral fit.

Spectral deconvolution of the amide I region was used to quantify the SF secondary structure for each processing condition [17] (Figure $4 \mathrm{~b}-\mathrm{d}$ ). The average values of the SF secondary structure's content are listed with their standard deviation in Table 3. After surface thiolation, the decrease of both the $\beta$-sheet and $\beta$-turn content can be observed, from $34.09 \%$ and $17.44 \%$ to $29.96 \%$ and $13.82 \%$, 
respectively, which corresponded with an increase in random coil from 29.67 to $37.31 \%$. This result indicates that grafting GSH molecules onto the SF film surface induced changes in the secondary structure of the silk fibroin, by converting the $\beta$-sheet and $\beta$-turn to random coil. Upon grafting BUD molecules onto the GSH-SF film through a thiol-ene click reaction, BUD-SF had the highest content of random coil, with $42.15 \%$, and the $\beta$-sheet content decreased to $25.65 \%$, while the $\beta$-turn content also decreased slightly to $12.19 \%$. The reduced $\beta$-sheet content in these spectra indicates that the $\beta$-sheet conformation of the silk fibroin was hampered throughout the grafting processes.

Table 3. Band assignments in FTIR spectra for each type of secondary structure within the amide I region of SF, and the relative ratio of SF secondary structure content for various films.

\begin{tabular}{cccc}
\hline Assignment & WA-SF (\%) & GSH-SF (\%) & BUD-SF (\%) \\
\hline $\begin{array}{c}\text { Side chains } \\
\left(1598-1605 \mathrm{~cm}^{-1}\right) \\
\text { Silk II, } \beta \text {-sheet }\end{array}$ & $0.13 \pm 0.23$ & $0.07 \pm 0.10$ & $0.01 \pm 0.01$ \\
$\left(1610-1635 \mathrm{~cm}^{-1}, 1695-1700 \mathrm{~cm}^{-1}\right)$ & $34.09 \pm 4.37$ & $29.96 \pm 2.69$ & $25.65 \pm 2.16$ \\
$\quad \begin{array}{l}\text { Random coil } \\
\left(1635-1645 \mathrm{~cm}^{-1}\right) \\
\text { Silk I, Type II } \\
\beta \text {-turn } \\
\left(1647-1654 \mathrm{~cm}^{-1}\right) \\
\alpha \text {-helix } \\
\left(1658-1664 \mathrm{~cm}^{-1}\right)\end{array}$ & $29.67 \pm 4.95$ & $37.31 \pm 4.01$ & $42.15 \pm 2.01$ \\
$\begin{array}{c}\text { Turns and bends } \\
\left(1666-1695 \mathrm{~cm}^{-1}\right)\end{array}$ & $17.44 \pm 1.84$ & $13.82 \pm 2.11$ & $12.19 \pm 1.55$ \\
& $10.40 \pm 1.95$ & $11.19 \pm 0.66$ & $11.96 \pm 0.52$ \\
& $8.28 \pm 0.84$ & $7.69 \pm 0.39$ & $8.05 \pm 0.62$ \\
\hline
\end{tabular}

\section{Conclusions}

In this study, we developed and assessed a new strategy for modifying silk fibroin film through thiol-ene click chemistry. Considering the myriad of alkene-containing molecules that have been adapted for thiol-ene click chemistry, this methodology makes it possible to introduce required functionalities at the SF film surface, and therefore can open up more opportunities for applications of the silk fibroin film in its device-based construction.

Supplementary Materials: The following are available online at http://www.mdpi.com/2227-9717/8/5/498/s1, Figure S1: Representative XP survey spectra of GSH-SF films exposed to neat BUD under $365 \mathrm{~nm}$ UV illumination for (a) 5 min and (b) $60 \mathrm{~min}$, Table S1: Bromine-to-carbon ratios of GSH-SF films covered with neat BUD and illuminated with $365 \mathrm{~nm}$ UV light for (a) $5 \mathrm{~min}$ and (b) $60 \mathrm{~min}$.

Author Contributions: X.Z. (Xiaoning Zhang) conceived the study, was in charge of administration and funding acquisition; X.Z. (Xiaoning Zhang) and J.L. determined the methodology; J.L., X.Z. (Xiaoning Zhang), Z.C., X.Z. (Xiaolin Zhang) and C.D. performed the experiments; the data was curated by X.Z. (Xiaoning Zhang), J.L., Z.C., X.Z. (Xiaolin Zhang) and C.D.; X.Z. (Xiaoning Zhang), J.L. and Z.C. wrote the original draft; the draft was reviewed and edited by X.Z. (Xiaoning Zhang), J.L. and G.C. All authors have read and agreed to the published version of the manuscript.

Funding: This research was funded by Venture \& Innovation Support Program for Chongqing Overseas Returnees grant number cx2019098; Chongqing Municipal Commission of Commerce grant number CQ2019JSCC03; Chongqing Graduate Student Research Innovation Project grant number CYS19121; Southwest University Faculty Start-up Fund grant number SWU117036. Chongqing Research Program of Basic Research and Frontier Technology grant number cstc2017jcyjAX0055; Part of this work was performed at the Chapel Hill Analytical and Nanofabrication Laboratory, CHANL, which receives support from NSF through the National Nanotechnology Coordinated Infrastructure, NNCI, program grant number ECCS-1542015.

Conflicts of Interest: The authors declare no conflict of interest. 


\section{Nomenclature}

$\begin{array}{ll}\mathrm{Na}_{2} \mathrm{CO}_{3} & \text { Sodium carbonate } \\ \mathrm{Na}_{2} \mathrm{HPO}_{4} & \text { Sodium phosphate dibasic dodecahydrate } \\ \mathrm{NaH}_{2} \mathrm{PO}_{4} \cdot 2 \mathrm{H}_{2} \mathrm{O} & \text { Sodium phosphate monobasic dihydrate } \\ \mathrm{CaCl}_{2} & \text { Calcium chloride } \\ \mathrm{C}_{2} \mathrm{H}_{5} \mathrm{OH} & \text { Ethanol } \\ \mathrm{H}_{2} \mathrm{C}=\mathrm{CH}\left(\mathrm{CH}_{2}\right)_{8} \mathrm{CH}_{2} \mathrm{Br} & \text { 11-bromo-1-undecene } \\ \mathrm{CH}_{3}\left(\mathrm{CH}_{2}\right)_{9} \mathrm{CH}_{2} \mathrm{Br} & \text { 1-bromoundecane }\end{array}$

\section{References}

1. Kaplan, D.L.; Sears, R.; Vunjak-Novakovic, G.; Meinel, L. Silk Fibroin Materials and Use Thereof. Patents Number US8361617 B2, 12 June 2018.

2. Mcgill, M.; Coburn, J.M.; Partlow, B.P.; Mu, X.; Kaplan, D.L. Molecular and macro-scale analysis of enzyme-crosslinked silk hydrogels for rational biomaterial design. Acta Biomater. 2017, 63, 76-84. [CrossRef] [PubMed]

3. Huang, J.; Xu, Z.; Qiu, W.; Chen, F.; Meng, Z.; Hou, C.; Guo, W.; Liu, X.Y. Stretchable and heat-resistant protein-based electronic skin for human thermoregulation. Adv. Funct. Mater. 2020, 30, 1910547. [CrossRef]

4. Wang, C.; Xia, K.; Zhang, Y.; Kaplan, D.L. Silk-based advanced materials for soft electronics. Acc. Chem. Res 2019, 52, 2916-2927. [CrossRef] [PubMed]

5. Murphy, A.R.; Kaplan, D.L. Biomedical applications of chemically-modified silk fibroin. J. Mater. Chem. 2009, 19, 6443-6450. [CrossRef] [PubMed]

6. Tao, H.; Kaplan, D.L.; Omenetto, F.G. Silk materials-A road to sustainable high technology. Adv. Mater. 2012, 24, 2824-2837. [CrossRef] [PubMed]

7. Kim, E.; Koo, H. Biomedical applications of copper-free click chemistry: In vitro, in vivo, and ex vivo. Chem. Sci. 2019, 10, 7835-7851. [CrossRef] [PubMed]

8. Raynal, L.; Allardyce, B.J.; Wang, X.; Dilley, R.J.; Rajkhowa, R.; Henderson, L.C. Facile and versatile solid state surface modification of silk fibroin membranes using click chemistry. J. Mater. Chem. B 2018, 6, 8037-8042. [CrossRef] [PubMed]

9. Pickens, C.J.; Johnson, S.N.; Pressnall, M.M.; Leon, M.A.; Berkland, C.J. Practical considerations, challenges, and limitations of bioconjugation via azide-alkyne cycloaddition. J. Bioconjugate. Chem. 2018, 29, 686-701. [CrossRef]

10. National Research Council. Prudent Practices in the Laboratory: Handling and Disposal of Chemicals; National Academies Press: Washington, DC, USA, 1995; p. 102.

11. McKenas, C.G.; Fehr, J.M.; Liu, B.; Donley, C.L.; Lockett, M.R. Mechanistic insights into UV-initiated thiol-ene reactions on amorphous carbon films. J. Phys. Chem. C 2018, 122, 21854-21860. [CrossRef]

12. Ryu, S.; Kim, H.H.; Park, Y.H.; Lin, C.C.; Um, I.C.; Ki, C.S. Dual mode gelation behavior of silk fibroin microgel embedded poly(ethylene glycol) hydrogels. J. Mater. Chem. B 2016, 4, 4574-4584. [CrossRef] [PubMed]

13. Lawrence, B.D.; Pan, Z.; Weber, M.D.; Kaplan, D.L.; Rosenblatt, M.I. Silk film culture system for in vitro analysis and biomaterial design. J. Visualized. Exp. 2012, 62, 3646. [CrossRef] [PubMed]

14. Zhong, H.R.; Fang, Y.; Bao, H.; Wu, T.F.; Zhang, X.N.; Xu, S.; Zhu, Y. Preparation and properties of silk fibroin based bilayer dressing materials. J. Text. Res. 2020, 41, 13-19. [CrossRef]

15. Sofia, S.; McCarthy, M.B.; Gronowicz, G.; Kaplan, D.L. Functionalized silk-based biomaterials for bone formation. J. Biomed. Mater. Res. 2001, 54, 139-148. [CrossRef]

16. Sy Piecco, K.; Aboelenen, A.M.; Pyle, J.R.; Vicente, J.R.; Gautam, D.; Chen, J. Kinetic model under light-limited condition for photoinitiated thiol-ene coupling reactions. ACS Omega 2018, 3, 14327-14332. [CrossRef] [PubMed] 
17. Liu, Q.; Deng, C.-H.; Sun, N. Hydrophilic tripeptide-functionalized magnetic metal-organic frameworks for the highly efficient enrichment of N-linked glycopeptides. Nanoscale 2018, 10, 12149-12155. [CrossRef] [PubMed]

18. Lu, Q.; Hu, X.; Wang, X.; Kluge, J.A.; Lu, S.; Cebe, P.; Kaplan, D.L. Water-insoluble silk films with silk I structure. Acta. Biomater. 2010, 6, 1380-1387. [CrossRef] [PubMed] 